\title{
Los entornos sociales y su relación con el conflicto y la violencia escolar en las ciudades de Armenia y Manizales, Colombia*
}

\section{Social environments and their relation to conflict and school violence in the cities of Armenia and Manizales, Colombia \\ Os contextos sociais e a sua relação com o conflito e a violência escolar nas cidades de Armenia e Manizales na Colômbia}

\author{
Leonardo Alberto Vega-Umbasía** \\ Álvaro Alfonso Fernández-Gallego *** \\ Néstor Raúl Giraldo-Navia **** \\ Cómo citar: Vega.Umbasía, L.A., Fernández- \\ Gallego, A.A., Giraldo-Navia, N.R. (2017) Los \\ entornos sociales y su relación con el conflicto y \\ la violencia escolar en las ciudades de Armenia y \\ Manizales, Colombia. Sophia, 13(1):34-46.
}

\begin{abstract}
** Magíster en Territorio, Conflicto y Cultura, Antropólogo y Administrador Público. Docente Investigador Programa de Gerontología, Universidad del Quindío, líder Grupo de Investigación en Desarrollo Correo electrónico: leonardovega@ uniquindio.edu.co. ORCID: 0000-0002-4431-5149.

***Especialista, Antropólogo, Docente Investigador programa de Trabajo Social, Universidad del Quindío, integrante Grupo de Investigación en Desarrollo, Correo electrónico. afernandez@ uniquindio.edu.co. ORCID: 0000-0002-4156-8554
\end{abstract}

****Especialista, Gerontólogo. Integrante Grupo de Investigación en Desarrollo. Consultor independiente (Atlanta, USA). Correo electrónico: luar666@gmail.com

\section{Resumen}

Este artículo se propone describir cómo los entornos sociales (familiares, barriales y escolares) están asociados a la problemática de la violencia escolar en las instituciones educativas públicas de las ciudades de Manizales y Armenia. Con un enfoque cuantitativo, de diseño transversal y alcance descriptivo, se aplicaron 500 encuestas a estudiantes de grados sexto a once y 30 a docentes y coordinadores. Los resultados muestran que los entornos sociales residenciales y escolares tienen variaciones notables en las dos capitales. En Manizales los entornos vecinales representan una mayor exposición a la venta y consumo de sustancias psico-activas, barras o pandillas futboleras y a personal armado; en Armenia, sus entornos escolares presencian y tienen mayor influencia las dinámicas violentas o adictivas.

Palabras clave: Colombia, conflictos sociales, educación media, violencia, violencia escolar.

Abstract

This article aims to describe how social environments (family, neighborhoods and school) are associated with problems related to school violence in state educational institutions of the cities of Manizales and Armenia. With a quantitative approach, cross-sectional design and descriptive scope, 500 surveys were applied to students in grades sixth to eleventh, and 30 to teachers and coordinators. The results show that residential and school social environments have remarkable variations

El presente artículo es el resultado del proyecto de investigación titulado "Convivencia, conflicto y violencia escolar en las instituciones educativas públicas del Eje Cafetero: Manizales y Armenia en el año 2014”, el cual contó con la financiación de la Vicerrectoría de Investigaciones de la Universidad del Quindío. Código Proyecto: 681. 
in the two capitals. In Manizales, the neighborhood environments have a great exposure to psychoactive substances sales and consumption, soccer hooligan gangs and armed personnel; in Armenia, violent or addictive dynamics.

Keywords: Colombia, social conflicts, secondary education, violence, school violence.

\section{Resumo}

Este artigo se propõe descrever a forma em que os contextos sociais (familiares, vizinhais e escolares) estão associados à problemática da violência escolar nas instituições educativas públicas das cidades de Manizales e Armenia. Com uma abordagem quantitativa, de desenho transversal e alcance descritivo, 500 sondagens foram aplicadas a estudantes de sexto a décimo-primeiro ano, e a 30 professores e coordenadores. Os resultados evidenciam que os contextos sociais residenciais e escolares têm variações notáveis nas duas capitais. Em Manizales, os contextos de vizinhança representam uma maior exposição à venda e consumo de substâncias psicoativas, barras e gangues relacionadas com o futebol e pessoal armado; em Armenia, os contextos escolares têm maior influência das dinâmicas violentas ou aditivas.

Palavras- chave: Colômbia, conflitos sociais, ensino médio, violência, violência escolar.

\section{Introducción}

En medio de la marcada situación de violencia letal o lesionante que todavía experimenta Colombia, a pesar de las tendencias sostenidas a la baja, se ha venido difundiendo, a través de los medios de comunicación, una práctica ya tipificada y ampliamente conocida de maltrato y victimización entre pares. Se trata del conocido "bullying" o acoso escolar, que hasta la fecha ha dejado un número indeterminado de víctimas, perpetuando el fenómeno de agresividad dentro de las instituciones educativas.

Preocupa aún más, si se tiene en cuenta que la escuela es uno de los principales medios de socialización y que esta forma inapropiada de interacción entre niños, niñas y adolescentes provoca la parcialización de los grupos que se asocian para maltratar a otro, causando graves consecuencias tanto emocionales como físicas.

Así pues, las burlas, propagación de rumores, injurias, exclusión de grupo, manipulación, coacción, intimidación, basadas en el desequilibrio de fuerzas, en el que una de las partes no logra defenderse por sí sola, son las formas más frecuentes de bullying, el cual se ha venido reportando en los departamentos de Caldas, Risaralda y Quindío ${ }^{1}$ y, de manera más

1. En Colombia, estos tres departamentos conforman la región conocida como el Eje Cafetero. Inicialmente el proyecto se diseñó para ser desarrollado en las ciudades capitales; sin embargo, no se aplicaron las encuestas en las instituciones educativas de Pereira (Risaralda) porque no se contó con la autorización correspondiente de la Secretaría de Educación Municipal. De ahí que esta investi- específica, en las ciudades capitales, de lo cual se han presentado una serie de trabajos investigativos que se orientan a conocer este fenómeno.

Es así como se hace necesario un acercamiento riguroso y crítico a los entornos familiares, barriales y escolares y su relación con la problemática del conflicto y la violencia escolar en el Eje Cafetero, para diseñar estrategias que permitan frenar este fenómeno y actuar en defensa de la población escolarizada de las instituciones educativas públicas, con el fin de evitar desenlaces graves en sus vidas y su salud. Esta problemática no ha sido abordada de manera íntegra por las autoridades académicas y gubernamentales locales a quienes les correspondería hacerlo, afirmación que puede darse luego de una minuciosa revisión de los antecedentes que se realizó para la construcción de la presente investigación.

Precisamente sobre este punto, son pertinentes las apreciaciones de Zurita (2015) quien ha señalado, en un reciente estudio sobre la violencia escolar desde los medios de comunicación y el poder legislativo en México, que en los últimos años se ha extendido la idea de que la violencia en las escuelas es un problema de primer orden que no le compete exclusivamente a las escuelas ni a los sistemas educativos $\mathrm{y}$, en consecuencia, que para su prevención y erradicación son indispensables los esfuerzos de una amplia variedad de actores. Sin embargo, Zurita considera

gación presente solo los resultados para las ciudades de Manizales y Armenia. 
que la principal dificultad para dicho abordaje radica en que los discursos construidos intersubjetivamente por los propios actores involucrados muestran no solo diferentes concepciones y visiones del problema; sino, que llegan hasta posturas irreconciliables acerca de su definición y, sin duda, en las propuestas de intervención para alcanzar dichos objetivos (Zurita, 2015).

\section{Marco de referencia teórico-conceptual ${ }^{2}$}

Quizás hoy una de las más relevantes teorizaciones sobre conflicto hacia su expresión violenta es la de Johan Galtung (1999), orientado al núcleo de la irenología (la paz); con él se ha generalizado una perspectiva que involucra tres dimensiones: cultural, estructural y directa, e implica los dos niveles de un continuo de relación social cuando se desarrolla como se ha expuesto, en detrimento de otro (no de su neutralización como defensa o protección): El conflicto (objetivos incompatibles) en términos de violencia (causar daño), se expresa en dos niveles: directa y subyacente. La subyacente, en estructural y cultural. Importante en tanto permite y remite el evento conflictivo a violento a un más que mero accidente o irregularidad en la vida, y sí a una articulación que le da sentido y explica su constancia en el tiempo; ya se verá que esta es una característica en el nivel interpersonal del acoso, hostigamiento o "matoneo" en la escuela que Olweus, pionero, lograra establecer como signo central de este ámbito.

Con las críticas que se le imputan, incluida la de Galtung, la de la Organización Mundial de la Salud (OMS) es mucho más precisa en la naturaleza,

2. Entre los años 2011 a 2015 el Observatorio Social de la Facultad de Ciencias Humanas y Bellas Artes de la Universidad del Quindío, en la plataforma de conflicto y violencia, adelantó una sistematización y monitoreo de las relaciones sociales que derivan en alta tensión hacia expresiones violentas de conflictos en el departamento del Quindío, tales como: homicidios, violencia interpersonal, violencia intrafamiliar, suicidios y violencia sexual. Por tal motivo, y como punto de partida para el análisis de la convivencia, el conflicto y la violencia escolar, el proyecto se apoyó en los avances teórico-conceptuales generados por el observatorio. Sin embargo, dado que el propósito del observatorio abarca los múltiples niveles desde lo micro a lo macro en contextos diversos, desde los cuales, según sean los condicionantes (estructurales o de posición y presión, de modo genérico resueltos en entramados de comunidad o sociedad), o la de la más laxa en términos de los lazos sociales de la elección racional hasta llegar de modo claro a la subjetiva e intencional, aquí en el presente artículo, se asumirá lo puntual respecto del estudio. ámbito y consecuencias de las acciones que por sus características, se definen como violentas:

El uso deliberado de la fuerza física o el poder, ya sea en grado de amenaza o efectivo, contra uno mismo, otra persona o un grupo o comunidad, que cause o tenga muchas probabilidades de causar lesiones, muerte, daños psicológicos, trastornos del desarrollo o privaciones (OMS, s.f.).

Permite concretar el campo conceptual sin renunciar a los prolegómenos ni a las matrices desde donde pueden tener sentido (no justificación) el que aparece como un sinsentido de las relaciones sociales si se supera la sola perspectiva utilitaria, económica.

Análogamente la violencia escolar desde Olweus se presenta cuando se produce en el contexto escolar como una:

Conducta de persecución física o psicológica que realiza el alumno o alumna contra otro, al que elige como víctima de repetidos ataques. Esta acción, negativa e intencionada, sitúa a las víctimas en posiciones de las que difícilmente pueden salir por sus propios medios. La continuidad de estas relaciones provoca en las víctimas efectos claramente negativos: disminución de su autoestima, estados de ansiedad e incluso cuadros depresivos, lo que hace difícil su integración en el medio escolar y el desarrollo normal de los aprendizajes. (Olweus, citado por Defensor del Pueblo 1999: 25-26).

Esta definición hace hincapié en tres criterios para identificar el maltrato por abuso de poder: a) la intención de hacer daño (físico o psicológico), b) la reiteración de las conductas y c) el desequilibrio de poder que hace a la víctima impotente para salir de esa situación por sí sola. Debido a este tercer criterio se ha de considerar el maltrato como un acto cobarde: quienes lo hacen saben que seguramente saldrán ilesos, ya que la víctima se siente impotente para responder y es difícil que quienes lo observan lo comuniquen.

Estando siempre presentes estas características, el maltrato entre iguales por abuso de poder toma formas muy distintas que es preciso conocer para poder entender su naturaleza compleja y planificar las intervenciones educativas adecuadas, formas que no siempre resultan evidentes a los observadores del fenómeno, incluso a quienes lo estudian.

Así, a lo largo de casi tres décadas de estudio, el concepto ha ido ampliándose al concebirse la 
diferencia de poder no solo en términos físicos, sino también psicológicos o sociales y al incluir nuevos ejemplos de conductas, como las relacionadas con la exclusión social u ostracismo, más allá de las interacciones explícitamente agresivas (véanse Del Barrio, Gutiérrez, Barrios, van der Meulen y Granizo, 2005; Rigby, 2007; Smith, Morita, Junger-Tas, Olweus, Catalano y Slee, 1999; Sullivan, Cleary y Sullivan, 2005).

Entre tanto, González (1993), considera que la violencia en la escuela es aquella en que son víctimas los niños y jóvenes la cual se manifiesta en los ámbitos extra-escolares. Tiene como presupuesto la visión distorsionada de una relación de subordinación legitima de la mayoría de unos miembros a otros. Se fundamenta no solamente en los patrones de intolerancia y coerción propios del conjunto de la sociedad, sino también en métodos pedagógicos basados en la sanción como modalidad de aprendizaje y en una relación de autoridad vertical y jerarquizada de la cual se derivan la dependencia, la sumisión, el miedo, la agresividad, la violencia, la segregación, la discriminación.

A lo que Pintus (2005) agrega que es una manifestación que se da en el espacio de las relaciones humanas en el contexto de las instituciones educativas. Las consecuencias vivenciales de esta violencia son negativas, como sentirse lastimado, dañado, despreciado, menospreciado y disminuido.

Por su parte, Beltrán, Torrado y Vargas (2016) en una reciente publicación sobre el hostigamiento escolar en las instituciones educativas públicas de la ciudad de Bucaramanga (Colombia), han clasificado, de acuerdo con Chaux (2012) y Moreno (1998), la violencia escolar así:

El hostigamiento escolar forma parte de la violencia escolar, toda vez que ésta, comprende los actos de daño, agresión y amenaza intencionales y sistemáticos, que se manifiestan en diferentes formas y niveles, clasificados en seis categorías de comportamiento antisocial: disrupción en las aulas, problemas de disciplina (conflictos entre profesores y estudiantes), hostigamiento entre iguales (bullying), vandalismo y daños materiales, violencia física (agresiones, extorsiones, etc.) y acoso sexual (Chaux, 2012; Moreno, 1998, Citados por Beltrán, Torrado y Vargas, 2016:174).

\section{Materiales y métodos}

Este estudio se orientó desde un enfoque cuantitativo, de carácter no experimental, descriptivo y transversal. Para la recolección de la información primaria se emplearon dos encuestas estructuradas ${ }^{3}$, una para estudiantes y otra para docentes y coordinadores. La realización de la muestra para el proyecto tuvo dos etapas en donde se implementó un muestreo aleatorio simple en ambas para asegurar la representatividad del estudio.

En la primera etapa se seleccionó la muestra de colegios para las ciudades de Armenia y Manizales. Para este propósito, se calculó primero el tamaño de la muestra para la implementación de los instrumentos a estudiantes usando la fórmula:

$$
m=\frac{\frac{Z^{2} P Q}{d^{2}}}{1+\frac{\frac{Z^{2} P Q}{d^{2}}}{N}}
$$

donde cada término y su respectivo valor se muestra como sigue:

- $Z$ : Valor correspondiente al nivel de confianza del (95\%), para este caso el valor correspondiente fue .

- $\boldsymbol{P}$ : Probabilidad de que suceda el evento, la cual se toma como

- Q: Probabilidad de que no suceda el evento, cuyo valor es similar al propuesto para la probabilidad de que sí suceda, es decir, .

- $d$ : Margen de error del muestreo, el cual se toma del 0 .

- $\boldsymbol{N}$ : Tamaño de la población, que en el proyecto para esta etapa fue el total de instituciones educativas en las ciudades de Armenia y Manizales que contarán con más de 300 estudiantes en secundaria.

- $\boldsymbol{m}$ : Tamaño de la muestra.

Así, se calculó una muestra de 18 colegios para ambas ciudades, de donde se seleccionarían los colegios de manera proporcional al número de instituciones educativas que tuvieran cada una usando la probabilidad de selección:

3. Se consultaron y ajustaron los instrumentos utilizados en los estudios realizados por la Defensoría del Pueblo de España (2007), Dane (2011), Vergara (2012), González y Martínez (2012) y Lugo y Valencia (2013). 


$$
\boldsymbol{p}=\frac{\boldsymbol{m}}{\boldsymbol{N}}
$$

De este modo, para Armenia con un total de 24 instituciones educativas se seleccionaron 7 y para Manizales con 33 instituciones educativas se seleccionaron 11 .

La segunda etapa de muestreo se realizó similar al procedimiento mostrado anteriormente, pero ahora el tamaño de la población fue el número de estudiantes de secundaria que pertenecían a los colegios de ambas ciudades. La muestra determinada fue de 500 estudiantes, 249 en Armenia y 251 en Manizales.

Para los coordinadores y docentes la muestra se tomó de manera intencional y se seleccionaron tres colegios de cada ciudad, de acuerdo con los niveles obtenidos de violencia (alta, moderada y baja). En cada colegio se aplicó a un coordinador y cuatro docentes para un total de 30 encuestas.

\section{Resultados}

Se tuvieron en cuenta un conjunto de variables sociodemográficas de los estudiantes y de sus familias, de su entorno familiar, barrial y escolar. En los docentes se indagó sobre los factores de incidencia individual, familiar, social y cultural que incentivan o limitan el rol de estudiante agresor o de víctima. De igual manera, se indagó entre ellos por las causas familiares y sociales que pueden influir en los problemas de coexistencia de los estudiantes.

\section{Datos socio-demográficos}

Figura 1. Perfil sociodemográfico estudiantes de Armenia

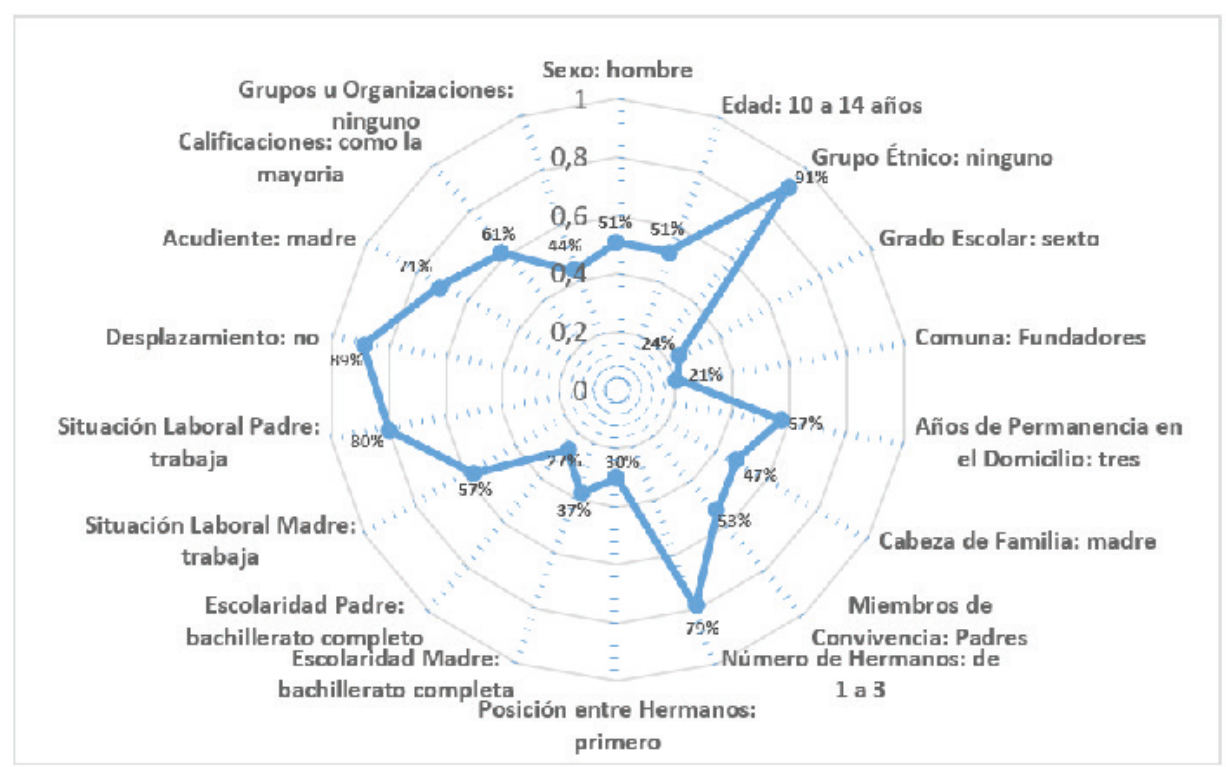

Fuente: Los autores 
Figura 2. Perfil sociodemográfico estudiantes de Manizales

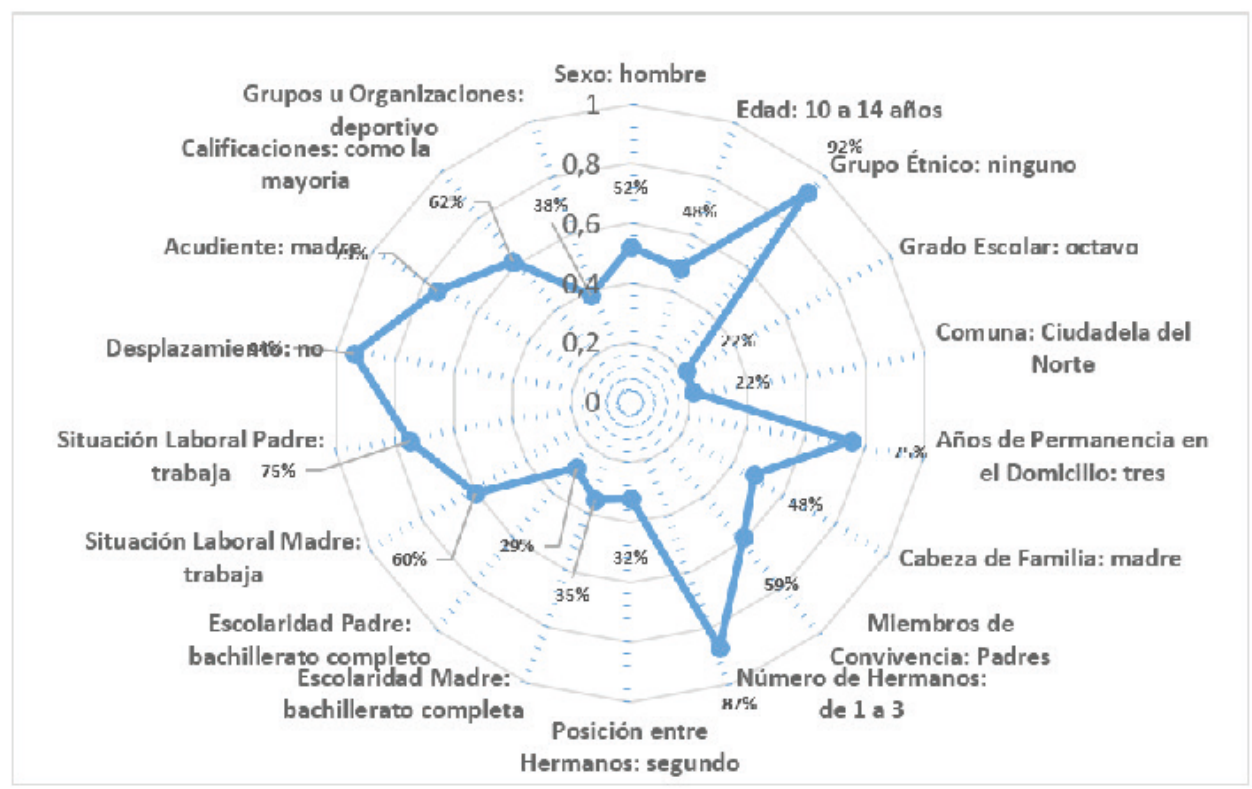

Fuente: Los autores

La edad no aparece significativa en general; sin embargo, hay 3 variaciones en los grupos: En la entrada, por las edades más bajas, Armenia, aunque menos del $1 \%$ tiene estudiantes de 10 años y con 11 años de edad un 9,2\%, mientras los de Manizales, en nivel de secundaria en las mismas edades su proporción es muy baja, con un $2,8 \%$. Lo que puede estar indicando un ingreso más prematuro al nivel de secundaria de los estudiantes de Armenia; entre 13 y 16 años Armenia tiene el 59,1\% y Manizales el 78\%; ya en el grupo de 17 a 20 años se nivelan con $14 \%$ Armenia y Manizales con 13,6\%.

Pertenencia étnica: Si bien la pertenencia étnica es uno de varios factores indicativos de migración inmigración en este caso-, para las capitales aludidas, la diferencia es casi nula en una y otra, a pesar de presentar el Quindío (con Bogotá, Meta y Risaralda) las tasa más altas de inmigración o de población no nativa - o no nacida en ella- residente en la ciudad, en una dinámica histórica mantenida. La población afro está entre 5 a $6 \%$, la raizal es equivalente a menos de $1 \%$. No informan el $1,6 \%$ y por el contrario de $91 \%$ a $92 \%$ son residentes nativos.

Con quién vive: siendo de respuesta múltiple, es decir, que se vive con los padres, pero además con otros familiares, por ejemplo, en Manizales hay una mayor presencia de los padres viviendo con sus hijos estudiantes: $59 \%$ frente a $53,4 \%$ de Armenia; por el contrario, en Armenia una fuente de socialización, como los abuelos es mucho más alta, $22.5 \%$ y en Manizales el $15.5 \%$. Es importante señalar que para las dos ciudades, una cuarta parte de los estudiantes viven solo con su madre.

En las dos ciudades, la mayor escolaridad es la de las madres de los estudiantes, tanto en bachillerato completo, como en estudios universitarios; la proporción más baja para las dos corresponde a sin ningún grado de estudio y en este caso es todavía menor para Manizales.

La situación laboral de los padres de los estudiantes mantiene al padre como el principal agente laboral de la familia, pero aun así, la proporción de madres trabajando es bastante alta, por encima del $50 \%$ de todas las madres con 57\% para Armenia, que se eleva a $60 \%$ para Manizales; buscando empleo están las madres en un 7,2\% en Manizales frente a un 3,6\% de Armenia, que reitera la orientación hacia el mercado del sexo femenino como mecanismo para sobrevivir el núcleo doméstico, además del padre.

En desplazamiento forzado, el Quindío como receptor más que expulsor de población, presenta el cuadro mayor de familias de estudiantes en esta situación -6\%-, duplicando a Manizales con 3\%.

El acudiente expresa también con quién viven los estudiantes que observamos al principio: los abuelos 
van desde 6\% en Manizales, a 9\% en Armenia; la madre sigue siendo la principal responsable escolar de los hijos con 71\% para Armenia y 75\% para Manizales y en segundo lugar los padres, pero en proporción 5 veces inferior a las madres.

\section{Entorno familiar}

\section{Manifestaciones de afecto por parte de los padres o acudientes}

Muy próximos entre sí, tanto los estudiantes de Armenia -93\%-, como los de Manizales -92\%expresan que reciben afecto, 6\% Armenia y 7\% Manizales poco afecto y un $1 \%$ para ambos, que nunca reciben afecto; dicen cosas buenas de los estudiantes en el $91 \%$ de Armenia y baja a $87 \%$ para Manizales; pocas veces $8 \%$ y $10 \%$ y se mantiene la franja de un $1 \%$ de estudiantes que son ignorados.

Lo que hacen los estudiantes es importante en $80 \%$ en Armenia y $84 \%$ en Manizales; pocas veces que es importante en $15 \%$ y $14 \%$ respectivamente. Nunca manifiestan que es importante lo que hacen de $2 \%$ a $4 \%$.

No reciben rechazo el $98 \%$ y $94 \%$ y al contrario entre casi siempre y siempre el $2 \%$ en Armenia y el $4 \%$ en Manizales son rechazados. Siempre o casi les dan confianza para que hablen de sus cosas 67\% en Armenia y 71\% en Manizales; mientras una proporción importante $23 \%$ y $21 \%$ pocas veces y nunca el 10\% Armenia y 7\% Manizales.

Ayudan a sus estudiantes cuando se sienten mal el 80\% en Armenia, el 83\% en Manizales; pocas veces la reciben el $13 \%$ para ambos conglomerados y nunca asciende a $6 \%$ en Armenia y 2\% en Manizales.

Saben dónde están los jóvenes y con quién salen entre el 87\% Armenia e igual para Manizales. Nunca 3\%.

Están enterados de cómo van en el colegio en el 92\% de los casos en Armenia y sin mayor diferencia, el $90 \%$ en Manizales.

\section{Conflictos dentro de la familia}

Amenazar a los jóvenes con echarlos de la casa o enviarlos lejos está en el 2\% para ambas capitales, mientras ofenderlos con términos despectivos de "bruto", "bobo" es más usual con un 15\% para las dos ciudades. Sacudones, pellizcos no reciben casi nunca o nunca el $94 \%$ de los estudiantes, pero sí de un $6 \%$ a $7 \%$, que se vuelve un $1 \%$ Armenia a $2 \%$ en Manizales, con trompadas, patadas y golpes corporales, y hasta un $3 \%$ con objetos.

Entre los propios padres de los estudiantes en disgustos llegan a insultos y amenazas de vez en cuando en un $28 \%$ en Armenia y asciende a $34 \%$ en Manizales; lo hacen habitualmente el $2 \%$ y a la violencia física corporal "de vez en cuando" entre un $12 \%$ en Armenia a $15 \%$ en Manizales, pero casi todos los días otra vez un $2 \%$ para los dos municipios.

En las interacciones de algunos miembros de la familia de los estudiantes, con otras personas distintas, el uso de armas en situaciones de tensión y conflicto entre un 5\% Armenia a un 7\% en Manizales de modo eventual las exhiben o usan directamente para herir y el $1 \%$ en las dos capitales lo hacen de forma habitual.

Que alguien de casa haya robado, para las dos un $10 \%$ lo ha hecho de vez en cuando y un $1 \%$ en las dos lo hace regularmente; en tanto ha empujado o golpeado a otros a veces para Armenia está en un alto $25 \%$ y para Manizales aún más alto de $33 \%$ y como conducta reactiva normalizada en Armenia un 2\% y en Manizales un 4\%.

Problemas derivados de consumo de alcohol, pocas veces, pero han ocurrido en Armenia en un 21\% y en Manizales en 24\% y de modo muy frecuente 5\% y $7 \%$ respectivamente, al igual problemas derivados del consumo de estupefacientes en un 9\% algunas veces en Armenia y en Manizales en un 7\% y con 3\% y $4 \%$ de manera continua por el consumo han tenido problemas; pero como reconocimiento de consumo solo la cifra asciende a $12 \%$ y $13 \%$ de consumidores ocasionales y de $6 \%$ de forma habitual en Armenia y Manizales.

\section{Abuso o acoso sexual a los estudiantes en el hogar}

El 2\%de los estudiantes de Armenia y el 3\% de Manizales revelan que tocan sus partes sexuales sin su consentimiento y el $1 \%$ que han intentado o los han forzado a relaciones sexuales.

Los estudiantes reconocen que algunos de sus compañeros usan armas para amenazar o herir en 25,5\% en Manizales y en Armenia un 18,5\%; han robado alguna vez para Manizales en 34\% y $18 \%$ Armenia y que algunos pertenecen a grupos que intimidan con 16\% en Manizales y $12 \%$ en Armenia, y que pertenezcan al mismo curso el 15,5\% Manizales, $12 \%$ Armenia. 
De los amigos expresan que algunos han golpeado a otra persona en $38 \%$ en Manizales y 34,5\% en Armenia.

El consumo de drogas una vez a la semana o el mes es equivalente en algunos amigos de los estudiantes en $27 \%$ Manizales y $23 \%$ Armenia. En consumo de bebidas embriagantes, aun siendo poco, la proporción aumenta para las dos capitales así: Manizales con $29 \%$, Armenia con $21 \%$.

Respecto de agresiones por grupos que amenazan, dentro del colegio la cifra aumenta respecto de amigos en general, con un muy alto 64\% Manizales y alto Armenia con $45 \%$.

El consumo de drogas y alcohol en el colegio es mayor para Armenia ahora con 23\% drogas y 7,2\% alcohol. En Manizales corresponde a 16,3\% drogas y aumenta a $13,5 \%$ el consumo de alcohol dentro de las instalaciones escolares.

La presión de los pares para la realización de acciones negativas se ha hecho sentir, aunque de media a baja escala y por una única vez, en $16 \%$ para estudiantes de ambas localidades para que golpeen a alguien, más de una vez en 9\% en Armenia y casi 12\% en Manizales.

En el manejo y control del cuerpo por parte del grupo de pares para sacarlos del límite de la intimidad y la privacidad personal, es en general respetuoso en más del 90\% en los dos municipios; tan solo, pero no por ello aceptable, confiesan ser presionados una vez para revelar sus partes íntimas un 3\% en Manizales y un $2 \%$ en Armenia, y de forma más reiterada, para las dos un $1,6 \%$.

\section{Factores de incidencia y causas}

Los docentes de los dos municipios consideran muy importante para limitar la acción agresiva, que exista poca contemplación con ella en la sociedad, al igual que sería contemplativa y casi cómplice la falta de disciplina escolar en la institución.

Respecto de las características de la personalidad y los problemas familiares del estudiante agresor, los docentes consideran que son decisivas según la escala de por lo menos $80 \%$ que les adjudican.

El entorno social residencial de los estudiantes barrio- divide la opinión de los docentes, que si bien lo consideran importante y aún muy importante superando el 50\% de las apreciaciones, lo es sin embargo menos que la familia y la misma personalidad del estudiante.

La extra-edad, y para la experiencia cotidiana de los docentes en su interacción con el colectivo escolar, aunque sea bienvenida como acceso a derechos, sí marca diferencias con los grupos de pares que simultáneamente son de edad similares.

Factores de riesgo que pueden incidir sobre la victimización y revictimización consideradas, no tienen un peso como el adjudicado para el estudiante agresor: Para el agredido y solo para los docentes de Armenia, la personalidad (poca aceptación propia, inseguridad) tiene un peso de $60 \%$, junto con las características de la familia del estudiante víctima o agredido y en este caso para los dos municipios en algo más del 50\% (53\%).

Las características físicas, étnicas, culturales o religiosas tienen peso, pero no son determinantes, y de menor rango las que menos afectan para ser o no agredido, los dos grupos de docentes consideran que las diferencias económicas.

A los factores familiares se les da un gran peso, a pesar de la variedad de procesos y agentes: desde la flexibilidad con el estudiante y su tiempo en distracciones visuales, hasta los desajustes de pareja; sin embargo, la permisividad con los estudiantes es mucho mayor en Manizales y en Armenia se considera el desajuste de pareja.

Como factor social general, ambiental, los dos grupos de docentes están de acuerdo que es el aumento de la violencia social, en tanto la violencia política es secundaria y aunque importante en Manizales el pertenecer a pandillas o "hinchadas" con el 53\%, y para Armenia es mucho más importante hasta alcanzar un $87 \%$.

Respecto de la influencia de los generadores de opinión, como los medios de comunicación, para los dos municipios los docentes consideran que internet y los personajes que sirven de modelos a los estudiantes.

En las relaciones de pares, la afirmación en el grupo aparece como objetivo superlativo en Manizales para los docentes al observar a sus estudiantes, mientras para los de Armenia se plantea que es la búsqueda de seguridad en sí mismo por parte del estudiante.

En general los docentes de los dos municipios consideran que el entorno espacial donde está la 
institución educativa es seguro; sin embargo, una proporción significativa percibe el entorno barrial de la I.E. inseguro: la tercera parte en Armenia y la cuarta para los docentes de Manizales.

En Manizales los entornos escolares como problema mayor está el de consumidores de estupefacientes o drogas en general, de expendios de droga, seguido de la presencia de "barras futboleras"; para los de Armenia el consumo y la venta de drogas es el problema mayor del entorno escolar, pero algunos puntos inferiores a sus homólogos de Manizales, mientras los grupos de aficionados no son tan perceptibles, pero sí y alto a muy alto los grupos de personas que amenazan.

\section{Discusión de resultados}

En el entorno familiar parece darse una correspondencia entre la consideración que merecen y obtienen los jóvenes (sobra decir de ambos sexos) en su hogar en términos afectivos, de reconocimiento, apoyo e interés por parte de sus padres o en su defecto de los acudientes en forma de indiferencia o de negación y rechazo, y las relaciones deterioradas, negativas generalizadas en el ámbito doméstico y de uso de la violencia y consumos de alcohol o estupefacientes; es decir, una franja pequeña pero siempre importante de los estudiantes no solo están expuestos a ambientes hostiles generalizados en su hogar; sino que además de un $1 \%$ a $2 \%$ sienten rechazo y negación en su territorio vital de protección y apoyo, y una franja más amplia asiste a relaciones (hasta un 15\% en Manizales y un poco pero no mucho menos, en Armenia) donde es posible y real, aunque no habitual, el desprecio, la ofensa y la agresión entre los miembros del hogar, al tiempo que los estupefacientes y el alcohol no son tampoco extraños como no lo es el delito (el robo a veces llega a 10\% y como modo de vida al $1 \%$ en los dos municipios capitales).

Los entornos sociales residenciales y escolares tienen variaciones notables en las dos capitales: mientras en Manizales los entornos vecinales representan una mayor exposición a la venta, consumo de spa; barras o pandillas futboleras y personal armado que en Armenia, aquí se invierte y será Armenia en sus entornos escolares donde se presenciarán y tendrán mayor influencia las dinámicas violentas o adictivas.

Dado que se trata del campo escolar, su institución formal es lugar constituyente de rivalidad y competencia, de conflicto agonístico, de sistemas de control e institucionalización, y de lógicas alternativas de anti control, que han sido esbozadas ya desde la racionalidad del panoptismo: vigilancia, jerarquización, división entre administradores y administrados. Reglas explícitas frente a lógicas alternativas y paralelas de intensidad variable.

Además, la escuela con su comunidad, es a su vez objeto espontáneo o planificado de: a). ofertas, o b), asedio, como clientela a la mano para el consumo, o para la generación y estímulo de nuevas necesidades para productos altamente rentables: los narco-consumos, la trata de personas, entre otros, por su gran significado de comunidad potencialmente disponible, gracias a sus características de concentrada, masiva, y estable, finalmente cautiva y accesible y de ello dan cuenta los promotores y vendedores de helados, forcha ${ }^{4}$, solteritas; la aparición de papelerías y fotocopiadoras, como la de vendedores de ilusiones y aventuras hasta los más racionales e inescrupulosos de explotación sexual o adictiva.

Los resultados presentados nos permiten analizar como los contextos territoriales de la institución escolar, así como los contextos territoriales (vecinales) de los escolares, son diferenciales de acuerdo con las lógicas de acción inscritas en sus tejidos sociales y que van desde áreas residenciales con cierto nivel de control y homogeneidad, hasta lugares de circuitos más abiertos y dinámicos. Hay además que contar con las trayectorias, dinámicas de calidad e intensidad de las relaciones domésticas que hacen de marco de referencia de los sectores que conforman la comunidad escolar.

En este foco la pluralidad de conceptos sobre un mismo término, sea violencia o conflicto generan imprecisión. La literatura es abundante en trabajos tanto empíricos como normativos, pero de modo necesario hay que cerrar el espectro de sentidos y pluralidad de significados sobre los que todavía hoy solo hay acuerdos parciales, debido a que el objeto mismo mantiene un gradiente de variabilidad, imprevisibilidad y azar en el tiempo: el de las relaciones sociales en perspectiva histórica y en el horizonte de los procesos de maduración y adaptación de la población escolar en particular: configuración de la identidad desde las matrices de la socialización y sus trayectorias, pero colmadas de las expectativas abiertas por la modernidad, y en consecuencia, de retos, oportunidades (menos o más amplias) y elecciones.

4. Bebida espumosa fermentada, elaborada con harina de trigo, especias y panela que se vende en las calles, muy típica de la región del Eje Cafetero. 
En juego está este "gradiente" o factor de modo simultáneo con las regularidades y ritmos desde los cuales se proyecta la vida institucional y social, y gracias a su mayor estabilidad se hace posible y perdurable y que se manifiesta en regularidades observables.

Este marco básico permite ir diferenciando procesos específicos por niveles, que generan altas cargas de tensión: los específicos de los procesos del desarrollo y maduración de la persona, ante sí y en la constitución de su yo en las relaciones sociales, con demandas contextuales personales, domésticas, de pares, barriales y escolares o institucionales, cercanas a lo que el funcionalismo descifra como roles. Estos procesos, observados ya no solo en los temporales de formación de la personalidad en sus relaciones; sino de modo sincrónico en las distintas posiciones, prescripciones y demandas anejas, se han denominado como "conflicto de roles", entrañan disyuntivas selectivas que en algún momento se vuelven excluyentes si se pretendiera asumirlas de forma simultánea y por lo cual se debe arriesgar una en detrimento de otra: No serían inherentemente conflictivas sino entrañaran una perdida, y aquí aparece ya un elemento diferenciador; sacrificar algo, una perdida que implica hasta cierto punto un daño, una falta y no simplemente una opción con sus riesgos y ganancias; sino el de la necesidad "imperativa" de renuncia, que es la entraña del conflicto de roles, donde la satisfacción o responsabilidad con uno implica la irresponsabilidad y el abandono en el otro, con daño, así sea reparable, pero que implica costo social y personal.

En esta perspectiva, el conflicto -intereses incompatibles o más leve, intereses y orientaciones divergentes- se puede situar por niveles, aunque sea posible articularlos a bases estructurantes (jerarquías sociales, normas y disenso, o de carácter mayor de estructurales al modo de Galtung): intraindividuales, interindividuales, colectivos e institucionales.

Lo que interesa, no es tanto la inherencia del conflicto en el desarrollo humano y las relaciones sociales, sino el tipo de conflicto que o desde el comienzo es negador o negativo o que puede derivar en la negación del otro, atenuada o radical, por acción u omisión.

En consecuencia, en las relaciones interpersonales, como es de suyo la dinámica en la comunidad educativa, atravesada por jerarquías, normas y posiciones, se plantea para poder abordarlo, al conflicto en sentido negativo y acudir a Max Weber nos puede cerrar para centrar el campo significativo de las relaciones de modo claro y conceptualmente riguroso, entendido como Poder; en los términos del autor: "Significa la probabilidad de imponer la propia voluntad dentro de una relación social, aun contra toda resistencia y cualquiera que sea el fundamento de esa probabilidad"(Weber, 2005:43). Refiriéndose al fundamento de ese poder, por lo amplio Weber lo denomina como amorfo para derivar hacia el de dominación que implica la probabilidad de obediencia a un mandato de determinado contenido.

El que el poder en los términos planteados deje un matiz, el de "aún contra toda resistencia", está planteando la posibilidad que la imposición de la voluntad no tropiece con resistencia, por los motivos que sea.

En nuestro interés específico, se destacan dos elementos: i) La imposición unilateral de la voluntad sobre otro (individual o plural) y ii) en una relación de desigualdad, o que la promueve.

Para plantear que el conflicto provocado en su inicio, o en su desarrollo, genera una relación de desigualdad y sometimiento que considera en la relación de manera preferente a exclusiva los intereses del lado del poder y la negación consiguiente del otro. Este conflicto es el que llamaremos negativo, dada la inmensa variación señalada no solo entre autores, sino entre situaciones y orientaciones (emocionales, utilitarias, culturales, ...). Intencionalmente intenta o genera menoscabo efectivo. Sin embargo, una de las características de algunos tipos de conflictos sociales culturalmente arraigados y justificados, y por lo tanto, inadvertidos por naturalizarse, sea por género, edad o estatus al ejercer el poder en detrimento de una de las partes, no se asumiría intencional en tanto no se reconoce como conflicto. Esta característica no solo no elimina el conflicto, sino que lo perpetúa a nombre de un estado de cosas, llámese tradición, corrección o educación.

Para mencionar algunos autores destacados por su trayectoria (se omiten muchos más como Feixas, del ámbito español), como F. Jiménez Bautista quien sitúa los conflictos en el horizonte de su propuesta de "paz neutra" en los siguientes planos que involucran desde lo micro a lo macro:

1.El lenguaje. $Y$ que es transversal a las relaciones sociales y a la ideación individual recogiendo a Habermas. 


\section{El ámbito personal.}

3. El ámbito familiar, diferentes intereses.

4. Todos los espacios no institucionales. "como, por ejemplo, los espacios públicos, escenarios de la vida cotidiana: el ámbito comunitario (el barrio)" (Jiménez, 2011:3).

Él mantiene, en la diversidad de conceptualizaciones, lo que se puede llamar como la base mínima común, o el consenso básico, al considerar que: "Los conflictos son procesos naturales que nos ayudan a clarificar intereses, percepciones, valores y que satisfacen necesidades, pasando a ser procesos creativos $\mathrm{y}$ constructivos que gradualmente deben aprender a controlar y a desarrollar al ser humano" (Jiménez, 20111: 23).

Por su parte, John Rex (1985), sociólogo británico, para decirlo de modo sumario (su propuesta es más amplia por supuesto), plantea que el conflicto empieza entre los objetivos del sujeto y las normas sociales, donde las acciones instrumentales o los objetivos del agente anteceden al de las normas. Esta razón sociológica en el tema es nodal: Las normas, aún en las comunidades más flexibles, como lo planteara Lucy Mair, no se cumplen automáticamente. Clave en el planteamiento es la disyunción entre intereses, vocaciones o valores de un agente y los de los sistemas normativos, que, en el ámbito escolar, o más ampliamente, en el institucional, así estén revestidos de autoridad, no por ello dejan de ser por lo menos incómodos.

\section{Conclusiones}

La violencia escolar es tratada como una categoría ambivalente y polisémica: pues no todo lo que sucede al interior de la institución educativa se refiere a violencias, ni tampoco puede decirse que allí no pasa nada violento. Además, debido a su polisemia esta categoría se ha vaciado en su contenido, de ahí el uso generalizado e inespecífico del término. A esto se suma la exclusión que dicha categoría ha tenido en la reflexión académica, aunque cabe aclarar, que el interés de conocimiento acerca de este fenómeno viene tomando fuerza desde las dos últimas décadas para el caso colombiano.

La información recolectada y procesada, y los resultados presentados llenan un vacío en el conocimiento existente sobre la coexistencia, el conflicto, y la violencia escolar dentro de las instituciones educativas de nuestra región y permite contrastar y, quizás, ampliar las teorías existentes sobre las causas y las consecuencias de la violencia escolar y todos los elementos que se ven involucrados alrededor de esta.

Los resultados de este ejercicio investigativo permiten identificar algunas alternativas necesarias para promover a nivel académico, la posibilidad de generar nuevos espacios que contribuyan a desarrollar estrategias de intervención dentro de las instituciones educativas; entendiendo estas, como potenciales espacios prácticos para la formación de futuros profesionales, beneficiando no solo la formación del individuo en su campo de acción y desarrollo de potencialidades, sino también generando programas y procesos que vayan en pro de la disminución de los índices de violencia.

Así mismo, es necesario considerar que los proyectos académicos para que sean eficaces, eficientes y trascendentales, deben estar ligados a la formulación de políticas públicas; por tanto, lo que se pretende es que, a partir de estos resultados presentados, se establezcan las condiciones necesarias para el inicio de la aplicación de unas estrategias de intervención estatal y académico y de una profunda y activa movilización social en las instituciones educativas de las ciudades del Eje Cafetero, que no solo mejore las relaciones de convivencia de quienes interactúan a diario en el entorno académico, sino también que el cambio de comportamiento y actitud se extiendan a los demás espacios donde los implicados se interrelacionan con otras personas, como son el contexto familiar y barrial.

Desde la presente investigación se plantea la posibilidad de que se construya un modelo de intervención, que no solo beneficie a la población estudiantil de básica primaria y secundaria públicas locales y regionales, sino que pueda extenderse a las demás regiones del país, complementando otros procesos que se están ejecutando actualmente y haciendo las modificaciones necesarias, según las necesidades propias del entorno donde se lleve a cabo, teniendo en cuenta, la vinculación de un organismo estatal como las correspondientes secretarias de educación que, como en este caso, facilitaron la ejecución del proyecto $\mathrm{y}$, de igual manera, son beneficiarias directas de los resultados obtenidos. 


\section{Referencias bibliográficas}

Beltrán, Y.; Torrado, O. y Vargas, C. (2016). "Prevalencia del Hostigamiento Escolar en las Instituciones Públicas de Bucaramanga-Colombia", Revista Sophia (Colombia), vol. 12, núm. 2, julio-diciembre, pp. 173-186.

Chaux, E. (2012). Educación, convivencia y agresión escolar, Bogotá: Taurus - Universidad de Los Andes.

Defensor del Pueblo de España. (1999). Informe del defensor del pueblo sobre violencia escolar. Disponible en: http://passthrough. fw-notify.net/download/268287/http:// www.conflictoescolar.es/wp-content/ uploads/2011/07/INFORME-DEL-DEFENSOR-DEL-PUEBLO-1999.pdf.

Defensor del Pueblo de España-UNICEF (2007). "Violencia Escolar: el maltrato entre iguales en la educación secundaria obligatoria 1999-2006". (Nuevo estudio y actualización del informe 2000). Disponible en: http:// www.oei.es/oeivirt/Informeviolencia.pdf.

Del Barrio, C., Gutiérrez, H., Barrios, A., van der Meulen, K. y Granizo, L. (2005). "Maltrato por abuso de poder entre escolares. ¿De qué estamos hablando?”. Disponible en: http://www.researchgate. net/publication/230628571_Del_Barrio_ Gutirrez_Barrios_van_der_Meulen Granizo_Maltrato_por_abuso_de_poder_ entre escolares_de qu_estamos_hablando.

Departamento Administrativo Nacional de Estadística-DANE- (2011). "Encuesta de convivencia escolar y circunstancias que la afectan para estudiantes de 50 a 11 o de Bogotá". Disponible en: http:// www.dane.gov.co/files/investigaciones/ boletines/educacion/formulario ConvivenciaEscolar_2011.pdf

Galtung, J. (1999). Tras la violencia, 3R: reconstrucción, reconciliación y resolución. Afrontando los efectos visibles e invisibles de la guerra y la violencia, Colección Red Gernika núm. 6, Bilbao: Bakeaz y Gernika Gogoratuz.
González, E. y Martínez, L. (2012). Convivencia, conflicto y violencia escolar en las instituciones educativas públicas en Armenia Quindío en el año 2012, Armenia: Universidad del Quindío. Tesis para optar el título de trabajadora social.

González, F. et al. (1993). Violencia en la Región Andina. El caso de Colombia, Bogotá: CINEP-APEP.

Jiménez, F. (2011). Racionalidad pacífica. Una Introducción de los Estudios para la Paz. Colección Paz y Conflictos, Madrid: Dykinson.

Moreno, J. (1998). "Comportamiento Antisocial en los Centros Escolares: Una Visión desde Europa", Revista Iberoamericana de Educación (España), núm. 18, septiembre-diciembre, pp.189-206.

Olweus, D. (1993). "La intimidación en la escuela: Lo que sabemos y lo que podemos hacer", Oxford: Blackwell Publishers. (Publicado en español en 1997 como Conductas de acoso y Amenaza Entre Escolares. Madrid: Ediciones Morata). Disponible en: http:// www.acosomoral.org/pdf/Olweus.pdf.

Organización Mundial de la Salud OMS (s.f.). "Informe mundial sobre la violencia y la salud". Sinopsis. Ginebra. Disponible en: http://apps.who.int/iris/ bitstream/10665/67411/1/a77102_spa.pdf.

Pintus, A. (2005). "Violencia en la escuela: compartiendo la búsqueda de soluciones", Revista Iberoamericana de Educación (España), núm.37, enero-abril, pp.117-34.

Rex, J. (1985). El conflicto social. Un análisis conceptual y teórico. Madrid: Siglo XXI Editores.

Rigby, K. (2007). "Bullying in schools and what to do about it". Disponible en:http:// www.educationengland.org.uk/articles/ rev04rigby.html.

Smith, P.; Morita, Y.; Junger-Tas, J.; Olweus, D.; Catalano, R. y Slee, P. (1999). The nature of school bullying: A cross-national perspective, London: Routledge. 
Sullivan, K.; Cleary, M. y Sullivan, G. (2005). Bullying en la enseñanza secundaria. El acoso escolar cómo se presenta y cómo afrontarlo, Barcelona: Ediciones CEAC.

Valencia, L. y Lugo, L. (2013). Convivencia, conflicto $y$ violencia escolar en las instituciones educativas privadas en Armenia Quindio en el año 2012, Armenia: Universidad del Quindío. Tesis para optar el título de trabajadora social.

Vergara, N. (2012). Convivencia, conflicto y violencia escolar en el primer semestre del año 2012, en la institución educativa Baudilio Montoya del municipio de Calarcá (Quindío), Armenia: Universidad del Quindío. Tesis para optar al título de Trabajadora Social.

Weber, M .(2005). Economía y sociedad. México: Fondo de Cultura Económica.

Zurita, U. (2015). "La violencia escolar desde los medios de comunicación y el poder legislativo en México". Revista Sophia (Colombia), vol. 11, núm. 1, enero-junio, pp. 81-93. 